\title{
Satisfaction with life amongst psychiatrists and general physicians: demographic and career variables
}

\author{
Yoram Barak $^{1 *}$, Moshe Tishler ${ }^{2}$, Dov Aizenberg ${ }^{3}$ \\ ${ }^{1}$ Abarbanel Mental Health Center, Bat-Yam, Israel; \\ ${ }^{2}$ Asaf-HaRofe Medical Center, Rishon LeZion, Israel; \\ ${ }^{3}$ Geha Mental Health Center, Petah-Tikva, Israel. \\ Email: ${ }^{*}$ mdybarak@Gmail.com
}

Received 1 July 2011; revised 12 August 2011; accepted 22 August 2011.

\begin{abstract}
Background: positive psychology and the science of happiness have impacted many realms in academia, including medicine. Recent reports from several countries suggest that physicians are unhappy with their profession. Aim: to assess levels of happiness amongst Israeli physicians and its' correlates. Method: participants in professional meetings endorsed the Satisfaction with Life Scale (SWLS) and a questionnaire detailing personal data (age, gender, marital status, number of children), specialty years as a physician and their state of health. Results: 223 physicians (131 psychiatrists, 92 primary care) completed the survey. The response rate was higher than $75 \%$. Mean SWLS score for all 223 physicians was $23.6 \pm$ 5.7 and did not differ between specialties' $(p=0.39$ ). No differences from reported norms amongst the general population were found. The only signifycant association with SWLS scores was number of children $(r=0.23 ; p=0.0006)$. Selection bias was inherent in this type of survey. Conclusion: satisfaction with life amongst Israeli physicians is equal to reported national means and may be supported by variables outside the professional sphere.
\end{abstract}

Keywords: Physicians; Satisfaction with Life; Happiness

\section{INTRODUCTION}

Happiness and satisfaction with life are two of the main constructs studied in the emerging field of positive psychology. Satisfaction with life is defined as "a person's cognitive and affective evaluations of his or her life" and denotes the presence of positive emotion, the absence of negative emotion, and a cognitive judgment of satisfaction and fulfillment [1]. The construct of happiness is an unwieldy term involving positive emotion and pleasure. Happy people have better quality of life, and research in the behavioral, social, and medical sciences is continuing to identify other benefits of happiness, including better health [2]. However some reports of doctors that are unhappy with their careers in medicine, with a significant minority of junior doctors considering leaving medicine have caused concern $[3,4]$. While most physiccians continue to report overall career satisfaction, only a small fraction of physicians are very satisfied with the practice of medicine [5].

Physician career satisfaction and morale has received focused attention recently. Publications deal with the growing discontent of physicians and attempt to unravel the causes underlying this attitude [6-8]. There are reasons for this attention. Physicians who are satisfied with their careers are likely to provide better health care than dissatisfied physicians. High physician satisfaction is also likely to result from good outcomes with patients. Moreover, dissatisfaction, if prolonged, may result in health problems for the physicians themselves [9].

The most obvious cause of doctors' unhappiness is that they feel overworked and undersupported [3]. Trained in pathophysiology, diagnosis, and treatment, doctors spend more time involved with issues like management, improvement, finance, law, ethics, and communication. Workload and pay, though important, do not fully explain the problem. A key factor seems to be a change in the psychological compact between the profession, employers, patients, and society so that the job is now different from what doctors expected [10]. On the other hand, the causes for physicians unhappiness herein mentioned might be partly offset by taking into account communication and ethical aspects in the primary education of medical students during recent years [11,12].

The last decade had witnessed an on-going struggle between the Israel Medical Association and governmental 
agencies focusing on the state of affairs of practicing medicine in an era of cutbacks and reform. As satisfaction with life reflects the cognitive judgment of one's happiness it will reveal insight into physicians' inner perception of their social and professional roles. Thus we aimed to evaluate the association between several career and demographic characteristics and satisfaction with life amongst physicians.

\section{METHOD}

The present study utilized the Satisfaction with Life Scale (SWLS) originally developed by Diener et al. [13]. The SWLS was developed to assess satisfaction with people's lives as a whole. The scale does not assess satisfaction with specific life domains, such as health or finances, but allows subjects to integrate and weigh these domains in whatever way they choose. The SWLS is a short, 5-item instrument designed to measure global cognitive judgments of one's lives. It takes only a few minutes to complete. The scale's items are: 1) In most ways my life is close to my ideal, 2) The conditions of my life are excellent, 3) I am satisfied with my life, 4) So far I have gotten the important things I want in life and 5) If I could live my life over, I would change almost nothing. Items are rated on a 1 - 7 basis as follows: 1-Strongly disgree, 2-Disgree, 3-Slightly disagree, 4-Neither agree nor disgree, 5-Slightly agree, 6Agree and 7-Strongly agree. The final score is the arithmetic total of all item scores reflecting satisfaction with life as follows: 35 - 31 Extremely satisfied, 26 - 30 Satisfied, 21 - 25 Slightly satisfied, 20-Neutral, 15 - 19 Slightly dissatisfied, 10 - 14 Dissatisfied and 5 - 9 Extremely dissatisfied $[14,15]$.

During the period Jan, 2007 to Dec, 2007 we presented the aims of the survey to physicians attending professional meetings. Regional meetings for the Israel Medical Association (IMA) family physicians, internists and psychiatric association were attended by one of the researchers. The Tel-Aviv chapter of the IMA holds a regional meeting every 4 months usually attended by 50 to 100 participants. Participants were than asked to complete the SWLS as well as a short questionnaire detailing personal data (age, gender, marital status, number of children), medical specialty years as a physician and the state of their health reflected in the following question: "Do you have medical problems that adversely affect your satisfaction with life?” This question was answered on the same 1 to 7 scoring as that of the SWLS items. Survey forms were handed to the participants and collected at the end of the meeting. Response rates were greater than $75 \%$ (ranging from $78 \%$ to $97 \%$ ).

We sampled various specialists: psychiatrists, general practitioners, internists working in general hospital set- tings and family physicians practicing in the community. There are 850 psychiatrists and 3000 primary care physicians registered with the IMA.

The study was approved by the local IRB.

\section{Statistical Analysis}

Data were analyzed using a paired-samples and independent-samples approach. The two-tailed t-test and non-parametric test were undertaken to test for differences between the evaluations for quantitative parameters. Examination of differences between the categorical parameters were based on the Pearson Chi-square and Fisher's Exact tests. All tests applied were two-tailed, and $\mathrm{p}$ value of $5 \%$ or less was considered statistically significant.

The data was analyzed using the Statistical Analysis System software, SAS Institute $6^{\text {th }}$ version, [16].

\section{RESULTS}

During the study period 223 physicians completed the survey. There were 131 psychiatrists and 92 primary care physicians. Mean age $( \pm$ SD) for the group was $52.3 \pm$ 11.5 years and there were 97 women and 126 men. The majority of participants, 182 (82\%) were married while there were 17 divorced, 11 widowed and 15 were single. There were 2.2 children per participant (median 2.0, range 0 to 7 ). On average the participants had been practicing their specialty for $21.5 \pm 11.7$ years (range: 6 46).

The mean total SWLS score for all 223 physicians was $23.6 \pm 5.7$ and did not differ significantly between the various specialties ( $p=0.39$ ). Categorically this translates to $84 \%$ of participants being "satisfied" with their lives (the sum of "slightly satisfied", "satisfied” and "very satisfied"). The SWLS total score was analyzed in order to identify correlations to demographic and clinical variables. Pearson correlation coefficients were calculated for: age, gender, number of children, years in practice and medical problems. The only statistically significant finding among all physicians $(n=223)$ was a linear correlation with the number of children $(r=0.23$; $p=$ 0.0006). There was an overall trend towards a positive correlation with increasing age $(p=0.06)$ reaching significance only for the psychiatrists' group $(\mathrm{r}=0.23$; $\mathrm{p}=$ 0.01).

The majority of physicians reported: "no medical problem that adversely affects satisfaction with life" (53\%). Medical problems "very much affecting" satisfaction with life were reported by a small minority of participants (4\%).

\section{DISCUSSION}

In the present study physicians from several specialties 
were surveyed and as a group reported mean levels of satisfaction with life equal to the Israel national mean for adults. In 2002 the government appointed the Bank of Israel to conduct a nationwide research as to happiness and satisfaction with life of Israel citizens [17]. The surveyors approached 9404 citizens 20 years of age and older. The majority of participants-83\% reported that they are "satisfied" with their lives from slightly satisfied to very satisfied. The questions used in this survey are similar to those presented in the SWLS. The level of such satisfaction with life was $84 \%$ in our sample completely in accord with the findings among the general population. The number of children was directly associated with increased level of satisfaction in physicians participating in the present study and in the general population of Israel [17]. Interestingly, while age was associated with decrease in life satisfaction in the general population it positively contributed to physicians' happiness. We may tentatively suggest that factors not related to career such as the number of children operate in the samples compared while older age indirectly reflects experience and possibly higher professional standing thereby contributing to physicians' satisfaction.

The satisfaction of medical specialists is affected by many variables such as family, stress, quality of services and professional rewards. Canadian researchers have compared psychiatrists to surgeons emphasizing that explanatory variables associated with their satisfaction with careers are among others intrinsic and extrinsic rewards and workload. In addition, their study demonstrated that variance associated with career satisfaction can be explained using various factors reported directly by physicians [18]. In Israel the IMA specifically interviewed a representative sample of 500 physicians focusing on professional satisfaction [19]. Only 59\% of physicians reported satisfaction with their work in this IMA survey. This again emphasizes that the high rates of satisfaction with life amongst physicians are influenced by extrinsic rewards such as children, religion and others in contrast to intrinsic service related variables $[15,20]$. These findings are supported by work undertaken in the USA evaluating the changing culture in modern medicine with special focus on the interactions and economic consequences of managed care, health service delivery systems, academic medicine, and psychiatry. In westernized medicine, especially the USA, improved technology and therapeutics profoundly impact medical culture; but medical economics is as significant. Physicians' roles blur with duties delegated to lesser qualified healthcare professionals to maximize patients seen and generated incomes Doctors need to understand that in entering medicine they enter a life's career in which they serve others, do not expect to become wealthy, but at life's end are able to state their satisfaction with their professional achievements [21,22].

Limitations of the present study include: 1) the data are self-reported; 2) the data are cross-sectional so that causal relations are subject to interpretation; 3) the sampling undertaken in professional academic meetings favors physicians who are possibly more socially and academically conscious and with a higher interest in their profession and therefore a higher satisfaction; 4) this sample is of small size only; 5) other specialties were not surveyed; 6) the SWLS does not specifically quantify satisfaction with ones' profession; and finally 7) no specific career related questions were posed. The SWLS scale reflects happiness indirectly. The association between career satisfaction and satisfaction with life may not be straight forward and we caution in overlapping these two concepts [23]. However, this is true for all survey data sets. Strengths of this study include: 1) easy to use measurement to assess global satisfaction; 2) high completion rate of surveys and 3) ability to compare date to a national study of satisfaction and happiness

In conclusion, satisfaction with life amongst Israeli physicians was very close to that of the general public. However, our findings suggest that happiness of physicians may be sustained by factors related to the personal and social domains unrelated to the professional sphere. The present small study sheds light on an important issue for all practicing physicians. It should be considered as a preliminary research and we aim to replicate this work with a much greater sample size and a variety of specialties.

\section{REFERENCES}

[1] Diener, E. and Seligman, M.E. (2002) Very happy people. Psychological Science, 13, 81-84. doi:10.1111/1467-9280.00415

[2] Lyubomirsky, S., King, L. and Diener, E. (2005) The benefits of frequent positive affect: Does happiness lead to success? Psychological Bull, 131, 803-855. doi:10.1037/0033-2909.131.6.803

[3] Smith, R. (2001) Why are doctors so unhappy? British Medical Journal, 322, 1073-1074. doi:10.1136/bmj.322.7294.1073

[4] Moss, P.J., Lambert, T.W., Goldacre, M.J. and Lee, P. (2004) Reasons for considering leaving UK medicine: Questionnaire study of junior doctors' comments. British Medical Journal, 329, 1263-1265. doi:10.1136/bmj.38247.594769.AE

[5] Mechanic, D. (2003) Physician discontent: Challenges and opportunities. Journal of the American Medical Assoiation, 290, 941-946. doi:10.1001/jama.290.7.941

[6] Chew, M. and Williams, A. (2001) Australian general practitioners: Desperately seeking satisfaction: Is the satisfied GP an oxymoron? Medicine Journal, 175, 85-86.

[7] Kassirer, J.P. (1998) Doctor discontent. New England 
Journal of Medicine, 339, 1543-1545. doi:10.1056/NEJM199811193392109

[8] Edwards, N., Kornacki, M.J. and Silversin, J. (2002) Unhappy doctors: What are the causes and what can be done? British Medical Journal, 324, 835-838. doi:10.1136/bmj.324.7341.835

[9] Sundquist, J. and Johannsson, S.E. (2000) High demand, low control, and impaired general health: Working conditions in a sample of Swedish general practitioners. Scand Journal of Public Health, 28, 123-131.

[10] Silversin, J. and Kornacki, M.J. (2002) Creating a physician compact that drives group success. Medicine Group Management Journal, 47, 54-62.

[11] Carufel-Wert, D.A., Younkin, S., Foertsch, J., Eisenberg, T., Haq, C.L., Crouse, B.J. and Frey, I.J.J. (2007) LOCUS: Immunizing medical students against the loss of professional values. Family Medicine, 39, 320-325.

[12] Kumagai, A.K. (2008) A conceptual framework for the use of illness narratives in medical education. Academic Medicine, 83, 653-658. doi:10.1097/ACM.0b013e3181782e17

[13] Diener, E., Emmons, R.A., Larson, R.J. and Griffin, S. (1985) The satisfaction with life scale. Journal of Personality Assess, 49, 71-75. doi:10.1207/s15327752jpa4901_13

[14] Pavot, W.G., Diener, E., Colvin, C.R. and Sandvik, E. (1991) Further validation of the satisfaction with life scale: Evidence for the cross-method convergence of well-being measures. Journal of Personality Assess, 57, 149-161. doi:10.1207/s15327752jpa5701_17

[15] Pavot, W. and Diener, E. (1993) Review of the satisfac- tion with life scale. Psychological Assess, 5, 164-172. doi:10.1037/1040-3590.5.2.164

[16] SAS/STAT Users' Guide (1990) Version 6, 4th Edition, Cary, NC, SAS Institute.

[17] Zusmann, N. and Romanov, D. (2004) Peoples happiness: Satisfaction with life of Israeli citizens. Research dept., bank of Israel. Jerusalem, Israel. http://www.boi.gov.il

[18] Lepnurm, R., Dobson, R., Backman, A. and Keegan, D. (2006) Factors explaining career satisfaction among psychiatrists and surgeons in Canada. Canada Journal of Psychiatry, 51, 243-255.

[19] Dgani, A. and Dgani, R. (2007) Satisfaction of Israeli physicians: Across several domains. GeoCartography. Israel medical association. http://www.ima.org.il

[20] Murray, A., Montgomery, J.E., Chang, H., Rogers, W.H., Inui, T. and Safran, D.G. (1997) Doctor discontent. A comparison of physician satisfaction in different delivery system settings, 1986 and 1997. Journal of General Internal Meddicine, 16, 452-459. doi:10.1046/j.1525-1497.2001.016007452.x

[21] Kaufman, K.R. (2000) Academic and private practice medicine and health service delivery systems: A psychiatric commentary. Annals Clinical Psychiatry, 12, 175177. doi:10.3109/10401230009147109

[22] Kaufman, K.R. (2008) The changing culture in modern medicine: A psychiatrist's perspective. The Open Social Science Journal, 1, 1-4. doi:10.2174/1874945300801010001

[23] Carrese, J.A. and Ibrahim, M.A. (2008) Success, regret, and the struggle for balance. Annals Family Medicine, 6, 171-172. doi:10.1370/afm.777 